\title{
PARTIALLY REDUCED FORMULATION OF SCALAR YUKAWA MODEL: POINCARÉ-INVARIANCE AND UNITARITY
}

\author{
I. Zahladko, A. Duviryak \\ Institute for Condensed Matter Physics of NAS of Ukraine, Lviv, UA-79011, Ukraine, \\ zagladko@icmp.lviv.ua,duviryak@icmp.lviv.ua
}

(Received February 12, 2012)

\begin{abstract}
We consider a scalar Yukawa-like model in the framework of partially reduced quantum field theory. The reduced Lagrangian of the model consists of free scalar field terms and nonlocal current interaction term. Hamiltonian expressions for conserved quantities arisen from a Lorentz-invariance of the model in the momentum representation have been found in the first-order approximation with respect to a coupling constant squared. The canonical quantization of the system is performed. It is shown that the obtained conserved quantities and the previously found Hamiltonian and momentum of the system satisfy the commutational relations of the Poincare group. The expression for $S$ matrix in the current approximation is found. The unitarity of this operator is proven by the direct calculation.
\end{abstract}

Key words: partially reduced Lagrangian, Yukawa model, Poincaré-invariance, unitarity.

PACS number(s): 11.10.Ef, 11.10.Lm, 11.30.Cp

\section{INTRODUCTION}

Recently a partially reduced field theory [1-4] complemented with the variational method $[5,6]$ has been used for the description of the relativistic bound states problem [7-11]. The structure of this approach is as follows. Variables of a field mediating an interaction of fermion or scalar matter fields are eliminated from the Lagrangian of the system by means of the covariant Green's function, the propagator of the mediating field. The reduced Lagrangian description is put into the Hamiltonian form which then is quantized canonically. Finally, a fieldtheoretical version of a variational method is applied in order to derive relativistic wave equations describing bound or/and scattering states of the system.

A reduced Lagrangian includes space-time-nonlocal interaction term. Unlike other nonlocal theories known in the literature where a nonlocality is inserted by hands (in a free-field Lagrangian $[12,13]$ or in interaction terms by means of form-factors [13, 14]), here the nonlocality appears in a natural way, via a propagator mediating an interaction between currents of matter field. Thus the reduced field theory describes those processes of the original local theory in which the role of free quanta of mediating field can be neglected. This approach has been used to the description of positronium (Ps), muonium $(\mathrm{Mu})[7,8] \mathrm{Ps}^{-}$and $\mathrm{Mu}^{-}[9]$, and the obtained spectra agree with the conventional QED and experimental data. The reduced scalar Yukawa model $[1,2,4,10]$ and its nonlinear generalizations [11] were considered too. In all cases it took sparing efforts to derive the variational wave equations of advantageous structure as compared with the Bethe-Salpeter (BS) equations.

However, some important problems of the partially reduced field theory remain unconsidered. A nonlocality of the Lagrangian complicates a transition to the Hamiltonian formalism. We apply a Hamiltonization scheme developed by Llosa and Vives [15] for nonlocal Lagrangians in mechanics. This procedure is realized by the subsequent approximation scheme and leads to a loss of covariance [4]. Non-covariance and non-exactness of this method cause to distrust in a relativistic invariance of the approach and thus in its physical meaningfulness. It is known that covariance is not a necessary condition of Poincaré-invariance of the system, but a Poincaréinvariance itself has a physical sense and it is a necessary condition of a reliability of results predicted by the theory.

In the present paper this problem is considered for the simple scalar Yukawa model. We construct the Hamiltonian formulation of a reduced Yukawa-like model in the linear (i. e., second-order coupling constant) approximation and prove a Poincaré-invariance of the model. Ten generators of the Poincaré group have been constructed for this purpose. They are built on the basis of Noether currents, by means of transition to the Hamiltonian formalism (Sections II-IV) and further quantization (Section V). It is worth mentioning that not only Hamiltonian but also boost generator contains the interaction term. It is shown that these generators satisfy commutational relations of the Poincaré group within the limits of the present approximation (Section VI).

One other important problem inherent to nonlocal field theories is the construction of a unitary scattering matrix. Usually, difficulties that arise herewith are the reasons to distrust such theories. In Subsection VII.A we construct the scattering matrix of the reduced Yukawa-like model by means of the standard quantummechanical algorithm [16], using a transition to the interaction representation. The unitarity of the scattering matrix within the present approximation is shown in Subsection VII.B. Some details of this computation are given there too.

We use the time-like Minkowski metrics: $\left\|\eta_{\mu \nu}\right\|=$ $\operatorname{diag}(+,-,-,-)$, and put $c=\hbar=1$. 


\section{REDUCED LAGRANGIAN AND CONSERVED QUANTITIES}

The considered model comes from the scalar Yukawa model [1] which describes the dynamics of two complex scalar fields $\phi_{r}(x),(r=1,2)$ coupled via a real scalar mediating field $\chi(x)$.

Reduction of the field $\chi(x)$ in the initial Lagrangian of the Yukawa model leads to an effective non-local Lagrangian describing the interaction of currents of fields $\phi_{r}(x)$ in terms of the symmetric Green function of KleinGordon equation [1,4]. For generality we replace the Green function by an arbitrary symmetric Poincaréinvariant kernel, $K\left(x-x^{\prime}\right)=K\left(x^{\prime}-x\right)$.

Hence a starting point of our work is a Lagrangain density:

$$
L=\sum_{r=1}^{2} L_{r}+\frac{1}{2} \int d^{4} x \rho(x) K\left(x-x^{\prime}\right) \rho\left(x^{\prime}\right),
$$

where

$$
\begin{gathered}
L_{r}=\left(\partial_{\mu} \phi_{r}^{*}\right)\left(\partial^{\mu} \phi_{r}\right)-m_{r}^{2} \phi_{r}^{*} \phi_{r}, \quad r=1,2, \\
\rho(x)=-\sum_{r=1}^{2} g_{r} \phi_{r}^{*} \phi_{r} .
\end{gathered}
$$

Poincaré-invariance of Yukawa model leads to the existence of ten conserved quantities which are a 4momentum $P^{\mu}$ and 4-angular momentum $M^{\lambda \sigma}$.

For Lagrangian (1) these expressions were found in [4]:

$$
\begin{aligned}
P^{\mu}(t) & =\left.\sum_{r=1}^{2} \int d^{3} x T_{r}^{0 \mu}(x)\right|_{x^{0}=t}-\left.\eta^{0 \mu} \int d^{3} x \int d^{4} x^{\prime} \rho(x) K\left(x-x^{\prime}\right) \rho\left(x^{\prime}\right)\right|_{x^{0}=t} \\
& -\frac{1}{2} \int d x^{4} \int d^{4} x^{\prime} \Xi\left(x^{0}-t, x^{\prime 0}-t\right) \rho(x)\left\{\partial^{\nu} K\left(x-x^{\prime}\right)\right\} \rho\left(x^{\prime}\right), \\
M^{\lambda \sigma}(t)= & \left.\sum_{r=1}^{2} \int d^{3} x T_{r}^{0[\lambda}(x) x^{\sigma]}\right|_{x^{0}=t}-\left.\int d^{3} x \int d^{4} x^{\prime} \rho(x) \eta^{0[\lambda} x^{\sigma]} K\left(x-x^{\prime}\right) \rho\left(x^{\prime}\right)\right|_{x^{0}=t} \\
& -\frac{1}{2} \int d x^{4} \int d^{4} x^{\prime} \Xi\left(x^{0}-t, x^{\prime 0}-t\right) \rho(x)\left\{\partial^{[\lambda} K\left(x-x^{\prime}\right) x^{\sigma]}\right\} \rho\left(x^{\prime}\right) .
\end{aligned}
$$

Here $a^{[\mu} b^{\nu]}=a^{\mu} b^{\nu}-a^{\nu} b^{\mu} \Xi(t, s) \equiv \Theta(t) \Theta(-s)-$ $\Theta(-t) \Theta(s)=\frac{1}{2}(\operatorname{sign}(t)-\operatorname{sign}(s))$, where $\Theta(t)-$ is the Heaviside step function, and

$$
T_{r}^{\mu \nu}=\left\{\left(\partial^{\mu} \phi_{r}^{*}\right)\left(\partial^{\nu} \phi_{r}\right)+\left(\partial^{\nu} \phi_{r}^{*}\right)\left(\partial^{\mu} \phi_{r}\right)\right\}-\eta^{\mu \nu} L_{r}
$$

is the energy-momentum tensor for a free field $\phi_{r}(x)$.

For further calculation it is convenient to transform each complex field into a pair of real fields: $\phi_{r \alpha}(x)$ $(r=1,2 ; \alpha=1,2)$ :

$$
\phi_{r}=\frac{1}{\sqrt{2}}\left(\phi_{r 1}+i \phi_{r 2}\right), \quad \phi_{r}^{*}=\frac{1}{\sqrt{2}}\left(\phi_{r 1}-i \phi_{r 2}\right),
$$

and, for brevity, we replace the multi subscript $r \alpha$ by single subscript $a(a=\overline{1,4})$. Then we have

$$
\rho(x)=-\frac{1}{2} \sum_{a} g_{a} \phi_{a}^{2}(x) .
$$

The transition to the Hamiltonian description was realized in [4] by means of the Hamiltonization procedure for nonlocal Lagrangians [15]. This transition is built as a perturbative scheme with the usage of the momentum representation for fields that in the first-order approximation (in a coupling constant squared) has the simple form:

$$
\begin{aligned}
& \phi_{a}(x)=\frac{1}{(2 \pi)^{3 / 2}} \sum_{A= \pm} \int \frac{d^{3} k}{\sqrt{2 k_{a 0}}} a_{a}^{A}(\mathbf{k}) e^{i A k_{a} x} \\
& a=1, \ldots, 4
\end{aligned}
$$

where $k=\left\{k_{a 0}, \mathbf{k}\right\}, k_{a 0}=\sqrt{m_{a}^{2}+\mathbf{k}^{2}}, \mathbf{k}=\left\{k^{i}, i=\right.$ $1,2,3\}$, and quantities $a_{a}^{A}$ are the amplitudes of normal field modes which upon quantization become the particles creation $(A=+)$ and annihilation $(A=-)$ operators.

For the generators of time translations $H=P^{0}$ (the Hamiltonian) and space translations $\mathbf{P}=\left\{P^{i}, i=\right.$ $1,2,3\}$ (the momentum) there were found the following expressions [4]:

$$
H=H_{\text {free }}+H_{\text {int }}, \quad \mathbf{P}=\mathbf{P}_{\text {free }},
$$

where

$$
\begin{aligned}
& H_{\text {free }}=\frac{1}{2} \sum_{a} \sum_{A} \int d^{3} k k_{a 0} a_{a}^{A}(\mathbf{k}) a_{a}^{-A}(\mathbf{k}), \\
& \mathbf{P}_{\text {free }}=\frac{1}{2} \sum_{a} \sum_{A} \int d^{3} k \mathbf{k} a_{a}^{A}(\mathbf{k}) a_{a}^{-A}(\mathbf{k}),
\end{aligned}
$$




$$
H_{\mathrm{int}}=\frac{1}{2} \sum_{a b} \sum_{A B C D} \int d^{3} k d^{3} q d^{3} u d^{3} v T_{a b}^{A B C D}(\mathbf{k}, \mathbf{q}, \mathbf{u}, \mathbf{v}) a_{a}^{A}(\mathbf{k}) a_{a}^{B}(\mathbf{q}) a_{b}^{C}(\mathbf{u}) a_{b}^{D}(\mathbf{v})
$$

and where

$$
T_{a b}^{A B C D}(\mathbf{k}, \mathbf{q}, \mathbf{u}, \mathbf{v})=-\frac{g_{a} g_{b}}{16(2 \pi)^{3}} \frac{\delta(A \mathbf{k}+B \mathbf{q}+C \mathbf{u}+D \mathbf{v})}{\sqrt{k_{a 0} q_{a 0} u_{b 0} v_{b 0}}} \widetilde{K}\left(A k_{a}+B q_{a}\right)
$$

$$
\widetilde{K}(k)=\int d^{4} x e^{-i k \cdot x} K(x) .
$$

In expressions (10) and (11) a summation over $A$ can be performed:

$$
\begin{aligned}
& H_{\text {free }}=\sum_{a} \int d^{3} k k_{a 0} a_{a}^{+}(\mathbf{k}) a_{a}^{-}(\mathbf{k}), \\
& \mathbf{P}_{\text {free }}=\sum_{a} \int d^{3} k \mathbf{k} a_{a}^{+}(\mathbf{k}) a_{a}^{-}(\mathbf{k}) .
\end{aligned}
$$

The translation generators $H$ and $\mathbf{P}$ must be supplemented with the generators of the Lorentz group for a further Poincaré-invariance examination of the system. These generators form into the 4-angular momentum.

\section{ANGULAR MOMENTUM}

\section{Zero-order approximation}

We find space components of the angular momentum in the zero-order approximation at first. The energymomentum tensor for complex scalar fields (6) must be substituted into the first term of (5). Thus we obtain the expression (with $i, j=1,2,3$ ):

$$
\begin{aligned}
M_{\text {free }}^{i j} & \equiv M_{(0)}^{i j}=\sum_{r} \int d^{3} x \\
& \times\left\{\dot{\phi}_{r}^{*}\left(x^{j} \partial^{i} \phi_{r}-x^{i} \partial^{j} \phi_{r}\right)+\dot{\phi}_{r}\left(x^{j} \partial^{i} \phi_{r}^{*}-x^{i} \partial^{j} \phi_{r}^{*}\right)\right\} .
\end{aligned}
$$

We proceed to real fields (7), rename subscripts $(r \alpha \rightarrow$ a), take into consideration representation (9), and obtain the components of the angular momentum vector:

$$
\begin{aligned}
M_{(0)}^{k} & \equiv \frac{1}{2} \varepsilon_{i j}^{k} M_{(0)}^{i j}=\frac{i}{2} \varepsilon_{i j}^{k} \sum_{a} \sum_{A} \int d^{3} k \\
& \times A a_{a}^{A}(\mathbf{k}) k^{i} \partial^{j} a_{a}^{-A}(\mathbf{k}), \quad k=1,2,3 .
\end{aligned}
$$

After summation over $A$ we have:

$$
M_{\text {free }}^{k} \equiv M_{(0)}^{k}=i \varepsilon_{i j}^{k} \sum_{a} \int d^{3} k a_{a}^{+}(\mathbf{k}) k^{i} \partial^{j} a_{a}^{-}(\mathbf{k})
$$

here $\partial^{i} a(\mathbf{k})=\partial a(\mathbf{k}) / \partial k_{i}$ etc.

\section{Fist-order approximation}

Using (5) and (6) the first correction for the angular momentum can be written as follows:

$$
\begin{aligned}
M_{(1)}^{i j} & =\int d^{4} x \int d^{4} x^{\prime} \Xi\left(x^{0}, x^{\prime 0}\right) \rho\left(x^{\prime}\right) K\left(x-x^{\prime}\right) \\
& \times\left[\partial^{i} \rho(x) x^{j}-\partial^{j} \rho(x) x^{i}\right] .
\end{aligned}
$$

Then we transform each complex field into a pair of real fields (7), take into consideration eqs. (8) and (9) and arrive at the formula:

$$
\begin{aligned}
M_{(1)}^{i j} & =4 i \sum_{a b} \sum_{A B C D} \int d^{3} k d^{3} q d^{3} u d^{3} v \\
& \times S_{a b}^{A B C D}(\mathbf{k}, \mathbf{q}, \mathbf{u}, \mathbf{v}) a_{a}^{A}(\mathbf{k}) a_{a}^{B}(\mathbf{q}) \\
& \times\left\{u^{j} a_{b}^{D}(\mathbf{v}) \partial^{i} a_{b}^{C}(\mathbf{u})-u^{i} a_{b}^{D}(\mathbf{v}) \partial^{j} a_{b}^{C}(\mathbf{u})\right. \\
& \left.+v^{j} a_{b}^{C}(\mathbf{u}) \partial^{i} a_{b}^{D}(\mathbf{v})-v^{i} a_{b}^{C}(\mathbf{u}) \partial^{j} a_{b}^{D}(\mathbf{v})\right\},
\end{aligned}
$$

where the kernel

$$
\begin{aligned}
& S_{a b}^{A B C D}(\mathbf{k}, \mathbf{q}, \mathbf{u}, \mathbf{v})=\frac{g_{a} g_{b}}{16(2 \pi)^{3}} \frac{\delta(A \mathbf{k}+B \mathbf{q}+C \mathbf{u}+D \mathbf{v})}{\sqrt{k_{a 0} q_{a 0} u_{b 0} v_{b 0}}} \\
& \times \mathcal{P} \frac{\widetilde{K}\left(A k_{a}+B q_{a}\right)-\widetilde{K}\left(C u_{b}+D v_{b}\right)}{A k_{a 0}+B q_{a 0}+C u_{b 0}+D v_{b 0}}
\end{aligned}
$$

was found in $[4]^{1}$.

\section{CENTRE-OF-MASS INTEGRAL}

\section{Zero-order approximation}

Similarly to the angular momentum we find an expression for the integral of centre-of-mass (it corresponds to pure Lorentz transformations) in zero-order approximation. For this purpose expression (6) for the energymomentum tensor must be substituted into the first term of (5) (where we assign $\lambda=0, \mu=i$ for superscripts):

\footnotetext{
${ }^{1}$ One of the authors (A.D.) asks pardon for an error made in Eq. (5.22) of Ref. [4] where the mistaken factor 1/16 is to be $\operatorname{read}$ as $1 / 8$.
} 


$$
K_{(0)}^{i} \equiv M_{(0)}^{0 i}=\sum_{r} \int d^{3} x\left\{2{\dot{\phi_{r}}}^{*} \dot{\phi}_{r} x^{i}-x^{0}\left(\dot{\phi}_{r}^{*} \partial^{i} \phi_{r}+\partial^{i} \phi_{r}^{*} \dot{\phi}_{r}\right)\right\} .
$$

After proceeding to real fields and some transformations and substitutions we obtain:

$$
K_{(0)}^{i}=\frac{i}{2} \sum_{a} \sum_{A} \int d^{3} k A a_{a}^{A}(\mathbf{k})\left\{k_{a 0} \partial^{i} a_{a}^{-A}(\mathbf{k})+\frac{k^{i}}{k_{a 0}} a_{a}^{-A}(\mathbf{k})\right\} .
$$

The second term contains two equal components with opposite signs. Thus, after summation over $A$, the only first term survives:

$$
K_{\text {free }}^{i} \equiv K_{(0)}^{i}=\frac{i}{2} \sum_{a} \int d^{3} k k_{a 0} a_{a}^{+}(\mathbf{k}) \stackrel{\leftrightarrow}{\partial^{i}} a_{a}^{-}(\mathbf{k})
$$

where $a \stackrel{\leftrightarrow}{\partial^{i}} b \equiv a \partial^{i} b-\left(\partial^{i} a\right) b$.

First-order approximation

According to (5), the centre-of-mass integral in the first-order approximation is:

$$
K_{(1)}^{i}=K_{\mathrm{int}}^{i}+K_{\mathrm{nc}}^{i},
$$

where

$$
\begin{aligned}
K_{\mathrm{int}}^{i}=-\frac{i}{2} \sum_{a b} \sum_{A B C D} A \int d^{3} k d^{3} q d^{3} u d^{3} v T_{b a}^{C D A B}(\mathbf{u}, \mathbf{v}, \mathbf{k}, \mathbf{q})\left\{\partial^{i} a_{a}^{A}(\mathbf{k})-\frac{k^{i}}{2 k_{a 0}^{2}} a_{a}^{A}(\mathbf{k})\right\} a_{a}^{B}(\mathbf{q}) a_{b}^{C}(\mathbf{u}) a_{b}^{D}(\mathbf{v}), \\
K_{\mathrm{nc}}^{i}=4 i \sum_{a b} \sum_{A B C D} \int d^{3} k d^{3} q d^{3} u d^{3} v S_{a b}^{A B C D}(\mathbf{k}, \mathbf{q}, \mathbf{u}, \mathbf{v}) a_{a}^{A}(\mathbf{k}) a_{a}^{B}(\mathbf{q}) \\
\times\left\{u_{b 0} a_{b}^{D}(\mathbf{v}) \partial^{i} a_{b}^{C}(\mathbf{u})+v_{b 0} a_{b}^{C}(\mathbf{u}) \partial^{i} a_{b}^{D}(\mathbf{v})+\frac{a_{b}^{D}(\mathbf{v}) a_{b}^{C}(\mathbf{u})}{2}\left(\frac{u^{i}}{u_{b 0}}-\frac{v^{i}}{v_{b 0}}\right)\right\} .
\end{aligned}
$$

\section{CHANGE OF VARIABLES. CANONICAL QUANTIZATION}

As was shown in [4], the variables $a_{a}^{+}, a_{a}^{-}$are noncanonical. We make a transition to canonical variables $\underline{a}$ that satisfy the Poisson bracket relations:

$$
\left\{\underline{a}_{a}^{-}(\mathbf{k}), \underline{a}_{b}^{+}(\mathbf{q})\right\}=i \delta_{a b} \delta(\mathbf{k}-\mathbf{q}) .
$$

(other Poisson brackets are equal to zero). These variables are related to the original ones by the approximated formula [4]:

$$
\begin{aligned}
a_{a}^{A}(\mathbf{k}) & =\underline{a}_{a}^{A}(\mathbf{k})+\frac{A}{2} \sum_{b} \sum_{B C D} \int d^{3} q d^{3} u d^{3} v \\
& \times S_{a b}^{-A B C D}(\mathbf{k}, \mathbf{q}, \mathbf{u}, \mathbf{v}) \underline{a}_{a}^{B}(\mathbf{q}) \underline{a}_{b}^{C}(\mathbf{u}) \underline{a}_{b}^{D}(\mathbf{v})+o\left(g^{2}\right),
\end{aligned}
$$

where the symbol $o\left(g^{2}\right)$ denotes terms of higher order than $g_{a}^{2}$ and $g_{a} g_{b}$. Thus the expressions for the angular momentum and for the centre-of-mass integral in terms of new variables $\underline{a}$ can be written as follows:

$$
\begin{aligned}
\mathbf{M} & =\mathbf{M}_{(0)}[a]+\mathbf{M}_{(1)}[a]=\mathbf{M}_{\text {free }}[a]+\mathbf{M}_{\mathrm{nc}}[a] \\
& =\mathbf{M}_{\text {free }}[\underline{a}]+o\left(g^{2}\right), \\
\mathbf{K} & =\mathbf{K}_{(0)}[a]+\mathbf{K}_{(1)}[a]=\mathbf{K}_{\text {free }}[a]+\mathbf{K}_{\text {int }}[a]+\mathbf{K}_{\mathrm{nc}}[a] \\
& =\mathbf{K}_{\text {free }}[\underline{a}]+\mathbf{K}_{\text {int }}[\underline{a}]+o\left(g^{2}\right) .
\end{aligned}
$$

Henceforth, for convenience, we do not underscore new variables, i. e. we rename $\underline{a} \rightarrow a$. Then the final expressions for the angular momentum and for the centre-ofmass integral in the momentum representation can be represented by formulae (19), (25) and (26). Together with (16), (15) and (12) this yields a dynamical basis for the system of two interacting scalar fields under consideration.

Let us perform the canonical quantization. Then the variables $a_{a}^{+}$are the creation operators and $a_{a}^{-}$are the annihilation operators. The normal ordering of products of these operators is understood. Poisson brackets should be replaced by quantum commutators:

$$
\{A, B\} \longrightarrow-i[A, B]
$$


For the operators $a^{ \pm}$standard commutational relations hold:

$$
\begin{aligned}
& {\left[a_{a}^{+}(\mathbf{k}), a_{b}^{+}(\mathbf{q})\right]=\left[a_{a}^{-}(\mathbf{k}), a_{b}^{-}(\mathbf{q})\right]=0,} \\
& {\left[a_{a}^{-}(\mathbf{k}), a_{b}^{+}(\mathbf{q})\right]=\delta_{a b} \delta(\mathbf{k}-\mathbf{q}) .}
\end{aligned}
$$

$$
\begin{aligned}
& {\left[P^{i}, H\right]=\left[P^{i}, H_{\text {free }}\right]+\left[P^{i}, H_{\text {int }}\right]=0,} \\
& {\left[M^{i}, H\right]=\left[M^{i}, H_{\text {free }}\right]+\left[M^{i}, H_{\text {int }}\right]=0,} \\
& {\left[P^{i}, P^{j}\right]=0, \quad\left[M^{i}, P^{j}\right]=i \varepsilon^{i j}{ }_{k} P^{k}, \quad\left[M^{i}, M^{j}\right]=i \varepsilon^{i j}{ }_{k} M^{k},} \\
& {\left[M^{i}, K^{j}\right]=\left[M^{i}, K_{\text {free }}^{j}\right]+\left[M^{i}, K_{\text {int }}^{j}\right]=i \varepsilon^{i j}{ }_{k} K^{k},} \\
& {\left[K^{i}, H\right]=\left[K_{\text {free }}^{i}, H_{\text {free }}\right]+\left[K_{\text {free }}^{i}, H_{\text {int }}\right]+\left[K_{\text {int }}^{i}, H_{\text {free }}\right]+o\left(g^{2}\right) \simeq i P^{i},} \\
& {\left[K^{i}, P^{j}\right]=\left[K_{\text {free }}^{i}, P^{j}\right]+\left[K_{\text {int }}^{i}, P^{j}\right]=i \delta^{i j} H,} \\
& {\left[K^{i}, K^{j}\right]=\left[K_{\text {free }}^{i}, K_{\text {free }}^{j}\right]+\left[K_{\text {free }}^{i}, K_{\text {int }}^{j}\right]+\left[K_{\text {int }}^{i}, K_{\text {free }}^{j}\right]+o\left(g^{2}\right) \simeq-i \varepsilon^{i j}{ }_{k} M^{k} .}
\end{aligned}
$$

It is easy to verify that commutation relations for freefield terms are valid. Let us present a calculation of commutation relations in the firs-order approximation in the coupling constant squared.

\section{A. Calculation of $\left[\mathbf{P}, H_{\mathrm{int}}\right]$ and $\left[M, H_{\mathrm{int}}\right]$}

Let us mention two remarks that concern to calculation of all commutators with interaction terms. The first concerns to normal ordering of products of the creation and annihilation operators. It is easy to verify that the normal ordering is preserved at every step of calculation below, regardless of the case how operators are ordered explicitly or not. Therefore for simplicity of the description we will to consider interaction terms of generators ordered implicitly. The second remark concern to superficial terms that arise during the calculation. Those terms will be omitted so far as they give a zero action in the Fock space.

Let us show the calculation of the first commutator in details here:

$$
\begin{aligned}
& {\left[\mathbf{P}, H_{\mathrm{int}}\right]=\frac{1}{2} \sum_{a b c} \sum_{A B C D} \int d^{3} p d^{3} k d^{3} q d^{3} u d^{3} v} \\
& \times T_{a b}^{A B C D}(\mathbf{k}, \mathbf{q}, \mathbf{u}, \mathbf{v}) \\
& \quad \times \mathbf{p}\left[a_{c}^{+}(\mathbf{p}) a_{c}^{-}(\mathbf{p}), a_{a}^{A}(\mathbf{k}) a_{a}^{B}(\mathbf{q}) a_{b}^{C}(\mathbf{u}) a_{b}^{D}(\mathbf{v})\right] .
\end{aligned}
$$

For brevity it is convenient to unify the integration variables $\mathbf{p}, \mathbf{k} \ldots$ and subscripts $a, b, c$ in the common sub- scripts $p, k, \ldots: a_{c}^{+}(\mathbf{p}) \equiv a_{p}^{+}, \ldots$ Let us consider a commutator in r.-h.s. of equality quoted above and execute some simplification in it. By consecutive permutations the operator product $a^{+} a^{-}$with operators $a_{k}^{A} \ldots a_{v}^{D}$ one obtains:

$\left[a_{p}^{+} a_{p}^{-}, a_{k}^{A} a_{q}^{B} a_{u}^{C} a_{v}^{D}\right]=-a_{k}^{A} a_{q}^{B} a_{u}^{C} a_{p}^{D} \delta_{p v} D$

$-a_{k}^{A} a_{q}^{B} a_{p}^{C} a_{v}^{D} \delta_{p u} C-a_{k}^{A} a_{p}^{B} a_{u}^{C} a_{v}^{D} \delta_{p q} B-a_{p}^{A} a_{q}^{B} a_{u}^{C} a_{v}^{D} \delta_{p k} A$.

Here we use the short notation: $\delta_{p k} \equiv \delta_{c a} \delta(\mathbf{p}-\mathbf{k})$ etc.

Integrating this expression and using some properties of $\delta$-function we receive:

$$
\begin{aligned}
& \int d^{3} p d^{3} k d^{3} q d^{3} u d^{3} v \mathbf{p}\left[a_{p}^{+} a_{p}^{-}, a_{k}^{A} a_{q}^{B} a_{u}^{C} a_{v}^{D}\right] \\
= & \int d^{3} k d^{3} q d^{3} u d^{3} v(A \mathbf{k}+B \mathbf{q}+C \mathbf{u}+D \mathbf{v}) a_{k}^{A} a_{q}^{B} a_{u}^{C} a_{v}^{D} .
\end{aligned}
$$

It is worth mentioning that expression (13) for $T_{a b}^{A B C D}$ contains a $\delta$-function. Thus we have

$$
\begin{aligned}
& \int d^{3} k d^{3} q d^{3} u d^{3} v(A \mathbf{k}+B \mathbf{q}+C \mathbf{u}+D \mathbf{v}) \\
& \times \delta(A \mathbf{k}+B \mathbf{q}+C \mathbf{u}+D \mathbf{v}) \cdots=0
\end{aligned}
$$

where the following property of $\delta$-function is taken into consideration: $\int d x \delta(x) x f(x)=0$ for arbitrary function $f(x)$ that is regular in $x=0$. Thus:

$$
\left[\mathbf{P}, H_{\text {int }}\right]=0 .
$$




\section{ZAHLADKO, A. DUVIRYAK}

The second commutator can be calculated by analogy. The terms that contain the derivative of $\delta$-function will occur there. In this case the differential operation should be displaced onto the one of operators $a^{ \pm}$(omitting the superficial terms). Finally, we obtain that the second commutator is equal to zero:

$$
\left[\mathbf{M}, H_{\text {int }}\right]=0 .
$$

\section{B. Calculation of $\left[H_{\text {free }}, \mathbf{K}_{\text {int }}\right]$ and $\left[H_{\text {int }}, \mathbf{K}_{\text {free }}\right]$}

Since free-field generators satisfy the Poincaré algebra (34) it is necessary to prove the equality:

$$
\left[H_{\text {free }}, \mathbf{K}_{\text {int }}\right]-\left[\mathbf{K}_{\text {free }}, H_{\text {int }}\right]=0 .
$$

In order to simplify calculations we proceed from operators $a^{ \pm}$to operators $b^{ \pm}$by means of the relation:

$$
a_{k}^{ \pm}=\sqrt{k_{0}} b_{k}^{ \pm} .
$$

In the new notation:

$$
\begin{gathered}
H_{\text {free }}=\int d^{3} k k_{0}^{2} b_{k}^{+} b_{k}^{-}, \\
\mathbf{K}_{\text {free }}=\frac{i}{2} \int d^{3} k k_{0}^{2} b_{k}^{+} \stackrel{\leftrightarrow}{\nabla} b_{k}^{-},
\end{gathered}
$$

$$
H_{\mathrm{int}}=\sum_{A B C D} \int d^{3} k d^{3} q d^{3} u d^{3} v \Pi_{k q u v}^{A B C D} b_{k}^{A} b_{q}^{B} b_{u}^{C} b_{v}^{D},
$$

$$
\mathbf{K}_{\mathrm{int}}=-i \sum_{A B C D} \int d^{3} k d^{3} q d^{3} u d^{3} v D \Pi_{k q u v}^{A B C D} b_{k}^{A} b_{q}^{B} b_{u}^{C} \nabla b_{v}^{D},
$$

(integrating by $d^{3} k d^{3} q d^{3} u d^{3} v$ include the summation over subscripts $a, b, c, d$ too; these subscripts are not showed explicitly in the formulae). Here the kernel $\Pi_{k q u v}^{A B C D}$ is similar by structure to the defined above kernel $T_{a b}^{A B C D}(\mathbf{k}, \mathbf{q}, \mathbf{u}, \mathbf{v})(13)$ :

$$
\begin{aligned}
\Pi_{k q u v}^{A B C D} & \equiv \Pi_{a b}^{A B C D}(\mathbf{k}, \mathbf{q}, \mathbf{u}, \mathbf{v})=\frac{1}{2} \sqrt{k_{a 0} q_{a 0} u_{b 0} v_{b 0}} T_{a b}^{A B C D}(\mathbf{k}, \mathbf{q}, \mathbf{u}, \mathbf{v}) \\
& =-\frac{g_{a} g_{b}}{32(2 \pi)^{3}} \delta(A \mathbf{k}+B \mathbf{q}+C \mathbf{u}+D \mathbf{v}) \widetilde{K}\left(A k_{a}+B q_{a}\right)
\end{aligned}
$$

Similarly to $T_{a b}^{A B C D}(\mathbf{k}, \mathbf{q}, \mathbf{u}, \mathbf{v})$ this kernel possesses symmetry properties:

$$
\begin{aligned}
\Pi_{a b}^{B A C D}(\mathbf{q}, \mathbf{k}, \mathbf{u}, \mathbf{v}) & =\Pi_{a b}^{A B C D}(\mathbf{k}, \mathbf{q}, \mathbf{u}, \mathbf{v}), \\
\Pi_{a b}^{A B C D}(-\mathbf{k},-\mathbf{q},-\mathbf{u},-\mathbf{v}) & =\Pi_{a b}^{-A-B-C-D}(\mathbf{k}, \mathbf{q}, \mathbf{u}, \mathbf{v})=\Pi_{a b}^{A B C D}(\mathbf{k}, \mathbf{q}, \mathbf{u}, \mathbf{v}), \\
\Pi_{a b}^{-A-B C D}(\mathbf{k}, \mathbf{q}, \mathbf{u}, \mathbf{v}) & =\Pi_{a b}^{A B C D}(\mathbf{k}, \mathbf{q},-\mathbf{u},-\mathbf{v}), \\
\Pi_{a b}^{A B-C D}(\mathbf{k}, \mathbf{q},-\mathbf{u}, \mathbf{v}) & =\Pi_{a b}^{A B C-D}(\mathbf{k}, \mathbf{q}, \mathbf{u},-\mathbf{v})=\Pi_{a b}^{A B C D}(\mathbf{k}, \mathbf{q}, \mathbf{u}, \mathbf{v})
\end{aligned}
$$

which are important for calculations. Summing up over $A, B, C, D$ in (39), (40) and using some properties (42) of $\Pi_{k q u v}^{A B C D}$ yields:

$$
\begin{aligned}
& H_{\mathrm{int}}=\int d^{3} k \ldots d^{3} v\left\{\Pi_{k q u v}^{---} b_{k}^{-} b_{q}^{-} b_{u}^{-} b_{v}^{-}+2\left(\Pi_{k q u v}^{+--}+\Pi_{u v k q}^{--+-}\right) b_{k}^{+} b_{q}^{-} b_{u}^{-} b_{v}^{-}\right. \\
& \left.+\left(\Pi_{k q u v}^{++--}+\Pi_{u v k q}^{--++}+4 \Pi_{k u q v}^{+-+-}\right) b_{k}^{+} b_{q}^{+} b_{u}^{-} b_{v}^{-}+2\left(\Pi_{k q u v}^{+++-}+\Pi_{u v k q}^{+-++}\right) b_{k}^{+} b_{q}^{+} b_{u}^{+} b_{v}^{-}+\Pi_{k q u v}^{++++} b_{k}^{+} b_{q}^{+} b_{u}^{+} b_{v}^{+}\right\}, \\
& \mathbf{K}_{\mathrm{int}}=i \int d^{3} k \ldots d^{3} v\left\{\Pi_{k q u v}^{----} b_{k}^{-} b_{q}^{-} b_{u}^{-} \nabla_{v} b_{v}^{-}+2 \Pi_{k q u v}^{+---} b_{k}^{+} b_{q}^{-} b_{u}^{-} \nabla_{v} b_{v}^{-}+\Pi_{u v k q}^{--+} b_{k}^{+} \nabla_{q} b_{q}^{-} b_{u}^{-} b_{v}^{-}\right. \\
& -\Pi_{u v k q}^{--+-} \nabla_{k} b_{k}^{+} b_{q}^{-} b_{u}^{-} b_{v}^{-}+\left(\Pi_{k q u v}^{++--}+2 \Pi_{k u q v}^{+-+-}\right) b_{k}^{+} b_{q}^{+} b_{u}^{-} \nabla_{v} b_{v}^{-}-2 \Pi_{k u q v}^{+-+-} b_{k}^{+} \nabla_{q} b_{q}^{+} b_{u}^{-} b_{v}^{-} \\
& -\Pi_{u v k q}^{--++} \nabla_{k} b_{k}^{+} b_{q}^{+} b_{u}^{-} b_{v}^{-}+\Pi_{k q u v}^{+++-} b_{k}^{+} b_{q}^{+} b_{u}^{+} \nabla_{v} b_{v}^{-}-\Pi_{k q u v}^{+++-} b_{k}^{+} b_{q}^{+} \nabla_{u} b_{u}^{+} b_{v}^{-}-2 \Pi_{u v k q}^{+-++} \nabla_{k} b_{k}^{+} b_{q}^{+} b_{u}^{+} b_{v}^{-} \\
& \left.-\Pi_{k q u v}^{++++} b_{k}^{+} b_{q}^{+} b_{u}^{+} \nabla_{v} b_{v}^{+}\right\} \text {. }
\end{aligned}
$$

Thereafter we calculate commutators for items with the fixed number of creation and annihilation operators separately. Let us calculate one of them.

It is worth mentioning at first the equalities:

$$
\left[b_{k}^{-}, b_{q}^{+}\right]=\frac{\delta_{k q}}{q_{0}},\left[\nabla_{k} b_{k}^{-}, b_{q}^{+}\right]=\frac{\nabla_{k} \delta_{k q}}{q_{0}},\left[b_{k}^{-}, \nabla_{q} b_{q}^{+}\right]=\frac{\nabla_{q} \delta_{k q}}{k_{0}} .
$$

Let us find commutator $H_{\text {free }}$ with the first term of expression for $\mathbf{K}_{\text {int }}(45)$ : 


$$
\int d^{3} p d^{3} k \ldots d^{3} v\left[p_{0}^{2} b_{p}^{+} b_{p}^{-}, i \Pi_{k q u v}^{---} b_{k}^{-} b_{q}^{-} b_{u}^{-} \nabla_{v} b_{v}^{-}\right]=i \int d^{3} k \ldots d^{3} v\left(k_{0}+q_{0}+u_{0}+v_{0}\right) \nabla_{v} \Pi_{k q u v}^{----} b_{k}^{-} b_{q}^{-} b_{u}^{-} b_{v}^{-},
$$

and commutator $\mathbf{K}_{\text {free }}$ with the first term of expression for $H_{\text {int }}(44)$ :

$$
\begin{aligned}
& \frac{i}{2} \int d^{3} p d^{3} k \ldots d^{3} v\left[p_{0}^{2}\left(b_{p}^{+} \nabla_{p} b_{p}^{-}-\nabla_{p} b_{p}^{+} b_{p}^{-}\right), \Pi_{k q u v}^{----} b_{k}^{-} b_{q}^{-} b_{u}^{-} b_{v}^{-}\right] \\
& =i \int d^{3} k \ldots d^{3} v\left(k_{0} \nabla_{k} \Pi_{k q u v}^{----}+q_{0} \nabla_{q} \Pi_{k q u v}^{----}+u_{0} \nabla_{u} \Pi_{k q u v}^{---}+v_{0} \nabla_{v} \Pi_{k q u v}^{---}\right) b_{k}^{-} b_{q}^{-} b_{u}^{-} b_{v}^{-} .
\end{aligned}
$$

Now it is necessary to show that the difference of integrals (47) and (48) is equal to zero, i. e.:

$$
\left(k_{0}+q_{0}+u_{0}+v_{0}\right) \nabla_{v} \Pi_{k q u v}^{----}-\left(k_{0} \nabla_{k} \Pi_{k q u v}^{----}+q_{0} \nabla_{q} \Pi_{k q u v}^{----}+u_{0} \nabla_{u} \Pi_{k q u v}^{----}+v_{0} \nabla_{v} \Pi_{k q u v}^{----}\right)=0 .
$$

To show this let us take into consideration the structure of kernel (41), namely

$$
\Pi_{k q u v}^{A B C D} \propto \delta(A \mathbf{k}+B \mathbf{q}+C \mathbf{u}+D \mathbf{v}) \widetilde{K}(A k+B q), \quad \text { where } \widetilde{K}(A k+B q) \equiv \widetilde{K}\left[(A k+B q)^{2}\right] .
$$

It is easy to approve the identity:

$$
k_{0} \nabla_{k} \widetilde{K}(k \pm q)+q_{0} \nabla_{q} \widetilde{K}(k \pm q)=0 .
$$

Expanding the expression in l.-h.s. of Eq. (49) yields:

$$
\begin{aligned}
& \left(k_{0}+q_{0}+u_{0}+v_{0}\right) \nabla \delta(\mathbf{k}+\mathbf{q}+\mathbf{u}+\mathbf{v}) \widetilde{K}(k+q)-k_{0} \nabla \delta(\mathbf{k}+\mathbf{q}+\mathbf{u}+\mathbf{v}) \widetilde{K}(k+q)-k_{0} \delta(\mathbf{k}+\mathbf{q}+\mathbf{u}+\mathbf{v}) \nabla_{k} \widetilde{K}(k+q) \\
& -q_{0} \nabla \delta(\mathbf{k}+\mathbf{q}+\mathbf{u}+\mathbf{v}) \widetilde{K}(k+q)-q_{0} \delta(\mathbf{k}+\mathbf{q}+\mathbf{u}+\mathbf{v}) \nabla_{q} \widetilde{K}(k+q)-u_{0} \nabla \delta(\mathbf{k}+\mathbf{q}+\mathbf{u}+\mathbf{v}) \widetilde{K}(k+q) \\
& -v_{0} \nabla \delta(\mathbf{k}+\mathbf{q}+\mathbf{u}+\mathbf{v}) \widetilde{K}(k+q)=-\delta(\mathbf{k}+\mathbf{q}+\mathbf{u}+\mathbf{v})\left(k_{0} \nabla_{k} \widetilde{K}(k+q)+q_{0} \nabla_{q} \widetilde{K}(k+q)\right)=0,
\end{aligned}
$$

thus equality (49) is true.

The commutators of terms with other rates of creation and annihilation operators can be calculated by analogy. Thus we have proved equality (35).

\section{Calculation of $\left[P^{i}, K_{\text {int }}^{j}\right]$ and $\left[M^{k}, K_{\text {int }}^{j}\right]$}

Upon calculating these commutators it is convenient to use the simplification proposed in the previous paragraph. Then the expressions for components of the momentum and the angular momentum are:

$$
P^{i}=\int d^{3} k p^{i} p_{0} b_{p}^{+} b_{p}^{-}
$$

and:

$$
M^{k}=i \varepsilon^{k}{ }_{i j} \sum_{a} \int d^{3} p p^{i} p_{0} b_{p}^{+} \partial^{j} b_{p}^{-} .
$$

After simple calculations we obtain:

$$
\left[P^{i}, K_{\mathrm{int}}^{j}\right]=i \delta^{i j} H_{\mathrm{int}} .
$$

As far as the second commutator is concerned, it is necessary to prove the equality:

$$
\left[M^{k}, K_{\mathrm{int}}^{l}\right]=i \varepsilon_{m}^{k l} K_{\mathrm{int}}^{m} .
$$

Let us show that this equality is fulfilled separately for items with fixed numbers of creation and annihilation operators in the expression $K_{\text {int }}^{j}$ (45). For example, for items with annihilation operators only we have:

$$
\begin{aligned}
& \varepsilon^{k}{ }_{i j} \int d^{3} p p^{i} p_{0} \Pi_{k q u \partial^{l} v}^{---}\left[b_{p}^{+} \partial^{j} b_{p}^{-}, b_{k}^{-} b_{q}^{-} b_{u}^{-} b_{v}^{-}\right] \\
& =-\varepsilon_{i j}^{k} \partial_{v}^{l} \Pi_{k q u v}^{---}\left(k^{i} \partial^{j} b_{k}^{-} b_{q}^{-} b_{u}^{-} b_{v}^{-}+q^{i} b_{k}^{-} \partial^{j} b_{q}^{-} b_{u}^{-} b_{v}^{-}\right. \\
& \left.+u^{i} b_{k}^{-} b_{q}^{-} \partial^{j} b_{u}^{-} b_{v}^{-}+v^{i} b_{k}^{-} b_{q}^{-} b_{u}^{-} \partial^{j} b_{v}^{-}\right) .
\end{aligned}
$$

Let us switch over the derivatives from the operators onto the kernel and throw away the unimportant superficial terms. Owing to antisymmetry of the factor $\varepsilon^{k}{ }_{i j}$ we receive the simple expression:

$$
\widehat{L}_{k} \partial_{v}^{l} \Pi_{k q u v}^{----} b_{k}^{-} b_{q}^{-} b_{u}^{-} b_{v}^{-},
$$

where $\widehat{L}^{k} \equiv \varepsilon^{k}{ }_{i j}\left(k^{i} \partial_{k}^{j}+q^{i} \partial_{q}^{j}+u^{i} \partial_{u}^{j}+v^{i} \partial_{v}^{j}\right)$ is the infinitesimal rotational operator. Let us write down:

$$
\widehat{L}^{k} \partial_{v}^{l} \Pi_{k q u v}^{----}=\partial_{v}^{l} \widehat{L}_{k} \Pi_{k q u v}^{----} b_{v}^{-}+\left[\widehat{L}_{k}, \partial_{v}^{l}\right] \Pi_{k q u v}^{----} .
$$

Since we have the rotary-invariant kernel the first term in r.-h.s. is equal to zero. After calculating the commutator $\left[\widehat{L}^{k}, \partial_{v}^{l}\right]=-\varepsilon^{k}{ }_{l j} \partial_{v}^{j}$, we present the sought expression 
(54) as:

$$
-\varepsilon_{i j}^{k} \partial_{v}^{j} \Pi_{k q u v}^{---} b_{k}^{-} b_{q}^{-} b_{u}^{-} b_{v}^{-} .
$$

It is easy to see that it gives the whole contribution in the r.-h.s. of (52) which contains annihilation operators only.

Commutators of the momentum with other lines of expression (45) for $K_{\text {int }}^{j}$ (that contain the creation operators too) can be found by analogy. Finally, we complete the proof of equality (52).
D. Calculation of $\left[K_{\text {free }}^{i}, K_{\text {int }}^{j}\right]$ and $\left[K_{\text {int }}^{i}, K_{\text {free }}^{j}\right]$

Let us find $\left[K_{\text {free }}^{i}, K_{\text {int }}^{j}\right]+\left[K_{\text {int }}^{i}, K_{\text {free }}^{j}\right]$

By analogy with the previous paragraph, we use expressions (38) and (45) for $K_{\text {free }}^{i}$ and $K_{\text {int }}^{j}$ in this sum in terms of $b^{ \pm}$operators. Then, using the commutational relations (46), both commutators are calculated line-byline.

For the first line of (45) the sum of commutators under consideration yields:

$$
\int d^{3} k \ldots d^{3} v\left\{\left(k_{0} \partial_{k}^{i} \partial_{v}^{j}+q_{0} \partial_{q}^{i} \partial_{v}^{j}+u_{0} \partial_{u}^{i} \partial_{v}^{j}+v_{0} \partial_{v}^{i} \partial_{v}^{j}-k_{0} \partial_{k}^{j} \partial_{v}^{i}-q_{0} \partial_{q}^{j} \partial_{v}^{i}+u_{0} \partial_{u}^{j} \partial_{v}^{i}-v_{0} \partial_{v}^{j} \partial_{v}^{i}\right) \Pi_{k q u v}^{---}\right\} b_{k}^{-} b_{q}^{-} b_{u}^{-} b_{v}^{-} .
$$

Taking into consideration the structure of kernel (50) and obvious equalities:

$$
\partial_{k}^{i} \partial_{v}^{j} \delta(\mathbf{k}+\mathbf{q}+\mathbf{u}+\mathbf{v}) \equiv \partial^{i} \partial^{j} \delta(\mathbf{k}+\mathbf{q}+\mathbf{u}+\mathbf{v})=\partial_{k}^{j} \partial_{v}^{i} \delta(\mathbf{k}+\mathbf{q}+\mathbf{u}+\mathbf{v})
$$

etc., we arrive at the expression:

$$
\begin{aligned}
& \int d^{3} k d^{3} q d^{3} u d^{3} v\left(k_{0} \partial_{v}^{j} \delta(\mathbf{k}+\mathbf{q}+\mathbf{u}+\mathbf{v}) \partial_{k}^{i} \tilde{K}(k+q)+q_{0} \partial_{v}^{j} \delta(\mathbf{k}+\mathbf{q}+\mathbf{u}+\mathbf{v}) \partial_{q}^{i} \tilde{K}(k+q)\right. \\
& -k_{0} \partial_{v}^{i} \delta(\mathbf{k}+\mathbf{q}+\mathbf{u}+\mathbf{v}) \partial_{k}^{j} \tilde{K}(k+q)-q_{0} \partial_{v}^{i} \delta(\mathbf{k}+\mathbf{q}+\mathbf{u}+\mathbf{v}) \partial_{q}^{j} \tilde{K}(k+q) b_{k}^{-} b_{q}^{-} b_{u}^{-} b_{v}^{-}
\end{aligned}
$$

that is equal to zero owing to (51). The other commutators of terms with definite rates of creation and annihilation operators can be calculated by analogy. Thus one receives:

$$
\left[K_{\text {free }}^{i}, K_{\text {int }}^{j}\right]+\left[K_{\text {int }}^{i}, K_{\text {free }}^{j}\right]=0 .
$$

\section{PROBLEM OF UNITARITY OF THE SCATTERING MATRIX}

\section{A. $S$-matrix construction}

For the construction of $S$-matrix we use the standard algorithm of quantum mechanics [16].
All the above mentioned quantities are given in Schrödinger representation, in which the field operators $a_{a}^{ \pm}(\mathbf{k}) \equiv a_{a}^{ \pm}(\mathbf{k}, t=0)$. Let us go over to the interaction representation. For this we write Heisenberg equation (relatively free-field Hamiltonian) for creating and annihilating operators:

$$
i \dot{a}_{a}^{A}(\mathbf{k}, t)=\left[a_{a}^{A}(\mathbf{k}, t), H_{\text {free }}\right] .
$$

We solve it and receive:

$$
a_{a}^{A}(\mathbf{k}, t) \equiv e^{i H_{\mathrm{free}} t} a_{a}^{A}(\mathbf{k}) e^{-i H_{\mathrm{free}} t}=e^{-i A k_{a 0} t} a_{a}^{A}(\mathbf{k}) .
$$

Using this expressions for calculating the interaction Hamiltonian we receive:

$$
\begin{aligned}
& H_{\text {int }}(t) \equiv e^{i H_{\text {free }} t} H_{\text {int }} e^{-i H_{\text {free }} t}=\frac{1}{2} \sum_{a b} \sum_{A B C D} \int d^{3} k d^{3} q d^{3} u d^{3} v T_{a b}^{A B C D}(\mathbf{k}, \mathbf{q}, \mathbf{u}, \mathbf{v}) \\
& \times e^{-i\left(A k_{a 0}+B q_{a 0}+C u_{b 0}+D v_{b 0}\right) t}: a_{a}^{A}(\mathbf{k}) a_{a}^{B}(\mathbf{q}) a_{b}^{C}(\mathbf{u}) a_{b}^{D}(\mathbf{v}):
\end{aligned}
$$

Let us consider an adiabatic scattering matrix in the first-order approximation:

$$
S=\lim _{\alpha \rightarrow 0} S_{\alpha}(\infty,-\infty) \equiv \lim _{\alpha \rightarrow 0} T e^{-i \int_{-\infty}^{\infty} d t e^{-\alpha|t|} H_{\mathrm{int}}(t)} \simeq 1-i \lim _{\alpha \rightarrow 0} \int_{-\infty}^{\infty} d t e^{-\alpha|t|} H_{\mathrm{int}}(t) ;
$$

here $T$ denotes the chronological ordering. Note that the operator of the chronological ordering $T$ does not play any role in the present approximation. Thus the calculation of $S$ is straitforward and the final expression for scattering matrix can be received:

$S=1+\frac{i}{2} \sum_{a b} \sum_{A B C D} \int d^{3} k d^{3} q d^{3} u d^{3} v \frac{g_{a} g_{b}}{16(2 \pi)^{3}} \frac{\delta^{(4)}\left(A k_{a}+B q_{a}+C u_{b}+D v_{b}\right)}{\sqrt{k_{a 0} q_{a 0} u_{b 0} v_{b 0}}} \widetilde{K}\left(A k_{a}+B q_{a}\right): a_{a}^{A}(\mathbf{k}) a_{a}^{B}(\mathbf{q}) a_{b}^{C}(\mathbf{u}) a_{b}^{D}(\mathbf{v}):$. 


\section{B. Checking for unitarity}

Now it should be shown that $S(55)$ is a unitary operator in the current approximation. It means:

$$
S S^{+} \approx 1+o\left(g^{2}\right) .
$$

The scattering matrix operator can be written as:

$$
S=I+i F,
$$

where $F$ up to the $i$ factor is equal to the transition operator. It matches in current approximation to a phase operator (following Blokhintsev; [14]). Accordingly to (56) $S$ is a unitary operator, if $F$ is Hermitian: $F=F^{+}$.
Let us show it is really so. For convenience we go over to the operators $b$ introduced in Subsection VI.B. Then

$$
\begin{aligned}
F & =\frac{1}{2} \sum_{a b} \sum_{A B C D} \int d^{3} k d^{3} q d^{3} u d^{3} v \\
& \times Q_{k q u v}^{A B C D}: b_{a k}^{A} b_{a q}^{B} b_{b u}^{C} b_{b v}^{D}:
\end{aligned}
$$

where $Q_{k q u v}^{A B C D}=\delta\left(A k_{a 0}+B q_{a 0}+C u_{b 0}+D v_{b 0}\right) \Pi_{k q u v}^{A B C D}$ is expressed here in terms of the kernel $\Pi_{k q u v}^{A B C D}$ (41) introduced in Subsection VI.B and possessing properties (42) and (43). In contrast to $\Pi_{k q u v}^{A B C D}$, the kernel $\mathrm{Q}$ satisfies properties (42) only.

After summation over $A, B, C, D$ in (57) we receive:

$$
\begin{aligned}
F & =\sum_{a b} \int d^{3} k \ldots d^{3} v:\left\{Q_{k q u v}^{----} b_{a k}^{-} b_{a q}^{-} b_{b u}^{-} b_{b v}^{-}+2 Q_{k q u v}^{+---} b_{a k}^{+} b_{a q}^{-} b_{b u}^{-} b_{b v}^{-}+2 Q_{u v k q}^{--+} b_{b k}^{+} b_{b q}^{-} b_{a u}^{-} b_{a v}^{-}\right. \\
& +Q_{k q u v}^{++--} b_{a k}^{+} b_{a q}^{+} b_{b u}^{-} b_{b v}^{-}+Q_{u v k q}^{--++} b_{b k}^{+} b_{b q}^{+} b_{a u}^{-} b_{a v}^{-}+4 Q_{k u q v}^{+-+-} b_{a k}^{+} b_{b q}^{+} b_{a u}^{-} b_{b v}^{-} \\
& \left.+2 Q_{k q u v}^{+++-} b_{a k}^{+} b_{a q}^{+} b_{b u}^{+} b_{b v}^{-}+2 Q_{u v k q}^{+-++} b_{b k}^{+} b_{b q}^{+} b_{a u}^{+} b_{a v}^{-}+Q_{k q u v}^{++++} b_{a k}^{+} b_{a q}^{+} b_{b u}^{+} b_{b v}^{+}\right\}:
\end{aligned}
$$

Hermiticity can be proved separately for some groups of terms in this expression: for the first and the last terms, for remaining terms of the first and the third line, separately Hermiticity for two first terms in the second line, and separately it can be shown for the third term in this line.

For example, we consider the sum of the first and the last terms in expression (58). Let us conjugate this sum:

$$
\begin{aligned}
& \left(Q_{k q u v}^{----}: b_{a k}^{-} b_{a q}^{-} b_{b u}^{-} b_{b v}^{-}:+Q_{k q u v}^{++++}: b_{a k}^{+} b_{a q}^{+} b_{b u}^{+} b_{b v}^{+}:\right)^{+} \\
& =Q_{k q u v}^{---}: b_{b v}^{+} b_{b u}^{+} b_{a q}^{+} b_{a k}^{+}:+Q_{k q u v}^{+++}: b_{b v}^{-} b_{b u}^{-} b_{a q}^{-} b_{a k}^{-}:
\end{aligned}
$$

In kernels $\mathrm{Q}$ we inverse all the signs $A, B, C, D$ accordingly to (42). Using normal ordering we order the operators $b$ by indices $k, q, u, v$ and receive the expression:

$$
Q_{k q u v}^{++++}: b_{a k}^{+} b_{a q}^{+} b_{b u}^{+} b_{b v}^{+}:+Q_{k q u v}^{---}: b_{a k}^{-} b_{a q}^{-} b_{b u}^{-} b_{b v}^{-}: .
$$

It coincides with an outgoing expression (in brackets in the left-hand side of (59)). Therefore the sum of the first and the last terms in (58) has the property of Hermiticity.

By analogy one can prove the hermiticity of other sums of lines in expression (58). At last we receive that $F$ is an Hermitian operator and it means that the scattering matrix $S$ is unitary.

\section{CONCLUSIONS}

We have considered the scalar Yukawa-like model within the framework of partially reduced field theory.
The Lagrangian of the model is a time-nonlocal functional, and a transition to the Hamiltonian formalism is a nontrivial problem. In the preceding work [4] the perturbative Hamiltonization procedure and a quantization of the model were proposed. This procedure leads to a loss of manifest covariance of the description. The question whether the procedure preserves a relativistic invariance of the model has remained open so far.

One of the aims of our work is a proof of the Poincaréinvariance of quantum Hamiltonian description of the reduced Yukawa-like model. For this purpose, the Hamiltonian counterparts to nonlocal Noether integrals of angular momentum and centre-of-mass of the system found earlier [4] have been built in the first-order approximation in a coupling constant squared $g^{2}$ and then have been quantized canonically. In order a Poincaréinvariance be guaranteed these operators together with the Hamiltonian and momentum of the system obtained in [4] must satisfy the commutation relations of Poincaré algebra, at least with the precision of up to $g^{2}$. This is indeed demonstrated in the paper.

Upon calculations of the commutators it wass been noticed that the availability or absence of a normal ordering in the expressions for generators does not influence the result of calculation of commutators of these generators. Moreover, the commutational relation remains preserved in the case when only separate items with the fixed number of creation and annihilation operators are retained in the interaction terms of canonical generators, (i. e., separate lines only in expressions (44) and (45)). It can be interpreted as if the Poincaré-invariance is held approximately on every finite sector of the Fock space of the model. It is expected also that the Poincaré-invariance is 


\title{
I. ZAHLADKO, A. DUVIRYAK
}

preserved in higher-order approximations in the coupling constant though this is difficult to realized even in the second-order (i.e., in $g^{4}$ ) approximation.

The other aim of our work concerns to scattering matrix for the investigated model. The explicit construction of S-matrix is realized and its approximate unitarity is proved. These results allow one to extend the field of application of this model to a scattering problem.

It is not surprising that the problem with unitarity does not arise in the present approach. The first reason is that we consider only lower-order approximations. The second reason is that the present model is close to the local Yukawa model rather than to typical models in the nonlocal field theory.

The investigation of more realistic systems such as the partially reduced spinor electrodynamics is addressed to the subsequent works.
[1] M. Barham, J. Darewych, J. Phys. A 31, 3481 (1998).

[2] J. Darewych, Condens. Matter Phys. 3, 633 (2000).

[3] V. Shpytko, J. Darewych, Phys. Rev. D 64, 045012 (2001).

[4] A. Duviryak, J. W. Darewych, J. Phys. A 37, 8365 (2004).

[5] L.I. Schiff, Phys. Rev. 130, 458 (1963).

[6] J. W. Darewych, Ukr. Fiz. Zh. 41, 41 (1996).

[7] J. W. Darewych, A. Duviryak, Phys. Rev. A 66, 032102 (2002).

[8] A. G. Terekidi, J. W. Darewych, J. Math. Phys. 45, 1474 (2004); A. G. Terekidi, J. W. Darewych, J. Math. Phys. 46, 032302 (2005)

[9] M. Barham, J. W. Darewych, J. Phys. B 41, 185001
(2008).

[10] M. Emami-Razavi, J. W. Darewych, J. Phys. G 31, 1095 (2005); M. Emami-Razavi, J. W. Darewych, J. Phys. G 32, 1171 (2006).

[11] A. Duviryak, J. W. Darewych, J. Phys. A 43, 485402 (2010).

[12] A. Pais, G. E. Uhlenbeck, Phys. Rev. 79, 145 (1950).

[13] G. V. Efimov, J. Part. Nucl. 35, 598 (2004).

[14] D. I. Blokhintsev, G. I. Kolerov, Nuovo Cim. A 44, 974 (1966).

[15] J. Llosa, J. Vives, J. Math. Phys. 35, 2856 (1994).

[16] A. S. Schwartz Mathematical foundations of the quantum field theory (Moscow, Atomizdat, 1975; in Russian).

[17] R. P. Gaida, Fiz. Elem. Chast. At. Yadra 13, 427 (1982).

\section{ЧАСТКОВО РЕДУКОВАНЕ ФОРМУЛЮВАННЯ СКАЛЯРНОЇ МОДЕЛІ ЮКАВИ: ПУАНКАРЕ-IHВАРІАНТНIСТЬ ТА УНIТАРНIСТЬ}

\author{
I. Загладько, А. Дувіряк \\ Інститут фізики конденсованих систем НАН України, \\ вул. Свенціцького, 1, Львів, 79011, Україна
}

\begin{abstract}
Ми розглядаємо скалярну модель типу Юкави в межах частково редукованої квантової теорії поля. Редукований лагранжіан складається з членів вільних скалярних полів та нелокальних членів взаємодії струмів. У першому наближенні за квадратом константи взаємодії знайдено імпульсне представлення гамільтонових виразів для збережуваних величин, що виникають внаслідок лоренц-інваріантності моделі. Здійснено канонічне квантування системи. Показано, що отримані збережувані величини і знайдені раніше гамільтоніан та імпульс системи задовольняють комутаційні співвідношення групи Пуанкаре. У цьому наближенні побудовано вираз для $S$-матриці. Прямими обчисленнями доведено унітарність цього оператора.
\end{abstract}

